\title{
An Inconsistency in the Spectrum of Bosonic Open 2-Brane
}

\author{
M. Abdul Wasay ${ }^{1,2}$ and Ding-fang Zeng ${ }^{1}$ \\ ${ }^{1}$ Institute of Theoretical Physics, Beijing University of Technology, Beijing 100124, China \\ ${ }^{2}$ Department of Physics, University of Agriculture, Faisalabad 38040, Pakistan
}

Correspondence should be addressed to M. Abdul Wasay; wasay_31@hotmail.com

Received 14 July 2015; Revised 3 October 2015; Accepted 7 October 2015

Academic Editor: Anastasios Petkou

Copyright (C) 2015 M. A. Wasay and D.-f. Zeng. This is an open access article distributed under the Creative Commons Attribution License, which permits unrestricted use, distribution, and reproduction in any medium, provided the original work is properly cited. The publication of this article was funded by SCOAP ${ }^{3}$.

We show that the spectrum of a bosonic open 2-brane does not contain any massless states to take the role of gravitons. Moreover, the spectrum of this open 2-brane only contains half integer mass squared values.

\section{Introduction}

Besides the impressive progress of string theories in the past few decades which compelled physicists to believe that a consistent theory of quantum gravity based on string philosophy would be built soon has slowed down. The early success of string theory also sparked the motivation to study higher dimensional extended objects. Indeed if one can give up 0-dimensional particles in favor of strings which are one-dimensional objects, then why not strings in favor of two-dimensional membranes? The basic idea that elementary particles could be interpreted as vibrating modes of a membrane originally came in 1962 by Dirac [1].

Since the task of achieving a consistent theory of quantum gravity based on string philosophy became more and more subtle, physicists tried to find alternative candidates to construct a theory of fundamental interactions; one of these candidates was supermembrane theory [2-4]. However, it was soon realized that the supermembrane has an unstable ground state [5].

Compared to the works on string quantization, works on membrane quantization are very few, precisely because of the difficulty the Nambu action meets when one tries to generalize it to $2+1$ dimensions or general $p+1$ dimensions. However, it is possible to quantize a bosonic membrane theory in a flat background $[6,7]$.

We will analyze the mass squared values in the spectrum of the bosonic open 2-brane. The bosonic membranes could be related to the bosonic string theory via dimensional reduction [8]. The critical spacetime dimension in analogy with bosonic string theory is taken to be $D=26$. We will focus on the spectrum of states after reviewing their quantization.

The organization of this paper is as follows: Section 2 is a brief review of the quantization scheme in a flat background [6]. Section 3 deals with the spectrum of this open 2-brane and Section 4 is summary and conclusion.

\section{A Brief Review}

The quantization of bosonic open branes was studied in $[6,7$, 9]. The open 2-brane dynamics is captured by the NambuGoto action, which physically describes the world-volume swept out by the brane. However, for quantization purposes it is not suitable because of the square root. However, one may follow [10] and write a Polyakov-type action for the general bosonic open 2-brane given by

$$
S=-\frac{1}{4 \pi \alpha^{\prime}} \int d^{3} \sigma \sqrt{-h}\left[h^{\alpha \beta} \partial_{\alpha} X^{\mu} \partial_{\beta} X_{\mu}+R-2 \Lambda\right],
$$

where $R$ and $\Lambda$ give the contribution from the cosmological constant term. A 2-brane sweeps out a 3-dimensional worldvolume which is parameterized by $\tau, \sigma^{1}, \sigma^{2}$. 
The energy-momentum tensor is given by the variation of the action (1), with respect to the world-volume metric $h^{\alpha \beta}$, as

$$
\begin{aligned}
T_{\alpha \beta} & =\frac{-2 \pi \alpha^{\prime}}{\sqrt{-h}} \frac{\delta S}{\delta h^{\alpha \beta}} \\
& =\partial_{\alpha} X^{\mu} \partial_{\beta} X_{\mu}-\frac{1}{2} h_{\alpha \beta} h^{\gamma \delta} \partial_{\gamma} X^{\mu} \partial_{\delta} X_{\mu}+\frac{1}{2} h_{\alpha \beta}=0 .
\end{aligned}
$$

Under the flat metric condition $h_{\alpha \beta}=\eta_{\alpha \beta}$, the components of this energy-momentum tensor can be written as

$$
\begin{aligned}
T_{\alpha \beta} & =\partial_{\alpha} X^{\mu} \partial_{\beta} X_{\mu}-\frac{1}{2} \\
\cdot & h_{\alpha \beta}\left[-\partial_{\tau} X^{\mu} \partial_{\tau} X_{\mu}+\partial_{\sigma^{1}} X^{\mu} \partial_{\sigma^{1}} X_{\mu}+\partial_{\sigma^{2}} X^{\mu} \partial_{\sigma^{2}} X_{\mu}\right] \\
& +\frac{1}{2} h_{\alpha \beta}
\end{aligned}
$$

with

$$
\begin{aligned}
& T_{00} \\
& =\frac{1}{2}\left[\partial_{\tau} X^{\mu} \partial_{\tau} X_{\mu}+\partial_{\sigma^{1}} X^{\mu} \partial_{\sigma^{1}} X_{\mu}+\partial_{\sigma^{2}} X^{\mu} \partial_{\sigma^{2}} X_{\mu}-1\right] \\
& =0 \text {, } \\
& T_{11} \\
& =\frac{1}{2}\left[\partial_{\tau} X^{\mu} \partial_{\tau} X_{\mu}+\partial_{\sigma^{1}} X^{\mu} \partial_{\sigma^{1}} X_{\mu}-\partial_{\sigma^{2}} X^{\mu} \partial_{\sigma^{2}} X_{\mu}+1\right] \\
& =0 \text {, } \\
& T_{22} \\
& =\frac{1}{2}\left[\partial_{\tau} X^{\mu} \partial_{\tau} X_{\mu}-\partial_{\sigma^{1}} X^{\mu} \partial_{\sigma^{1}} X_{\mu}+\partial_{\sigma^{2}} X^{\mu} \partial_{\sigma^{2}} X_{\mu}+1\right] \\
& =0 \text {, }
\end{aligned}
$$

and the Euler-Lagrange equation for the $X^{\mu}$ fields is given by

$$
\left(\partial_{\tau}^{2}-\partial_{\sigma_{1}}^{2}-\partial_{\sigma_{2}}^{2}\right) X^{\mu}\left(\tau, \sigma_{1}, \sigma_{2}\right)=0 .
$$

By imposing Neumann boundary condition

$$
\begin{aligned}
& \partial_{\sigma^{1}} X^{\mu}\left(\tau, 0, \sigma^{2}\right)=\partial_{\sigma^{1}} X^{\mu}\left(\tau, \pi, \sigma^{2}\right)=0, \\
& \partial_{\sigma^{2}} X^{\mu}\left(\tau, \sigma^{1}, 0\right)=\partial_{\sigma^{2}} X^{\mu}\left(\tau, \sigma^{1}, \pi\right)=0,
\end{aligned}
$$

one can get the following modes expansion for the $X^{\mu}$ fields:

$$
\begin{aligned}
& X^{\mu}\left(\tau, \sigma^{1}, \sigma^{2}\right)=\frac{x^{\mu}}{\sqrt{\pi}}+\frac{2 \dot{\alpha} p^{\mu}}{\sqrt{\pi}} \tau \\
& +i \sqrt{2 \dot{\alpha}} \sum_{m, n=0}^{+\infty}\left(n^{2}+m^{2}\right)^{-1 / 4} \\
& \cdot\left(X_{n m}^{\mu} e^{i \tau \sqrt{n^{2}+m^{2}}}-X_{n m}^{\dagger \mu} e^{-i \tau \sqrt{n^{2}+m^{2}}}\right) \cos n \sigma^{1} \cos m \sigma^{2}
\end{aligned}
$$

and the canonical momentum, as

$$
\begin{aligned}
P^{\mu}(\sigma)= & \frac{p^{\mu}}{\pi \sqrt{\pi}}+\frac{1}{\pi} \sqrt{\frac{2}{\alpha}} \sum_{m, n=0}^{+\infty}\left(n^{2}+m^{2}\right)^{1 / 4} \\
& \cdot\left(P_{n m}^{\mu \dagger} e^{i \tau \sqrt{n^{2}+m^{2}}}+P_{n m}^{\mu} e^{-i \tau \sqrt{n^{2}+m^{2}}}\right) \cos n \sigma^{1} \\
& \cdot \cos m \sigma^{2} .
\end{aligned}
$$

According to the standard commutation relation

$$
\left[X^{\mu}, P^{\nu}\right]=\eta^{\mu \nu} \delta\left(\sigma^{1}-\sigma^{\prime 1}\right) \delta\left(\sigma^{2}-\sigma^{\prime 2}\right)
$$

the following commutation relations between the creation/ annihilation operators can be obtained:

$$
\begin{aligned}
& {\left[X_{n m}^{\mu}, P_{n^{\prime} m^{\prime}}^{\nu}\right]} \\
& \quad=\frac{1}{\pi} \eta^{\mu \nu}\left[\delta_{n n^{\prime}} \delta_{m m^{\prime}}-\frac{1}{2} \delta_{-n, n^{\prime}} \delta_{m m^{\prime}}-\frac{1}{2} \delta_{n n^{\prime}} \delta_{-m, m^{\prime}}\right] .
\end{aligned}
$$

Using these creation/annihilation operators, the Hamiltonian of the system is expressed as

$$
\begin{aligned}
& 4 \pi \alpha^{\prime} H=\int_{0}^{\pi} d \sigma^{1} \int_{0}^{\pi} d \sigma^{2}\left(P_{\mu} \dot{X}^{\mu}-\mathscr{L}\right) \\
& =2 \alpha^{\prime} \pi^{2} \eta_{\mu \nu} \sum_{n=1}^{\infty} n\left[X_{n 0}^{\mu \dagger} X_{n 0}^{\nu}+X_{n 0}^{\mu} X_{n 0}^{\nu \dagger}\right] \\
& +2 \alpha^{\prime} \pi^{2} \eta_{\mu \nu} \sum_{m=1}^{\infty} m\left[X_{0 m}^{\mu \dagger} X_{0 m}^{\nu}+X_{0 m}^{\mu} X_{0 m}^{\nu \dagger}\right] \\
& +\alpha^{\prime} \pi^{2} \eta_{\mu \nu} \sum_{n, m=1}^{\infty}\left(n^{2}+m^{2}\right)^{1 / 2}\left[X_{n m}^{\mu \dagger} X_{n m}^{\nu}+X_{n m}^{\mu} X_{n m}^{\nu \dagger}\right] \\
& +4 \pi \alpha^{\prime 2} p^{2} .
\end{aligned}
$$

Under the tensorial assumption,

$$
\begin{aligned}
& X_{n m}^{\mu}=\sqrt{\frac{2}{\pi}} \phi_{n}^{\mu \dagger} \otimes \phi_{m}^{\mu \dagger}, \\
& X_{n m}^{\mu \dagger}=\sqrt{\frac{2}{\pi}} \phi_{n}^{\mu} \otimes \phi_{m}^{\mu},
\end{aligned}
$$

the Hamiltonian (11) is translated into

$$
H=N_{1}+N_{2}+N_{12}+a+b-\alpha^{\prime} M^{2},
$$


where

$$
\begin{aligned}
N_{1} & =\eta_{\mu \nu} \sum_{n=1}^{\infty} n\left\{\phi_{n}^{\mu \dagger} \otimes \phi_{0}^{\mu \dagger}\right\}\left\{\phi_{n}^{\mu} \otimes \phi_{0}^{\mu}\right\}, \\
N_{2} & =\eta_{\mu \nu} \sum_{m=1}^{\infty} m\left\{\phi_{0}^{\mu \dagger} \otimes \phi_{m}^{\mu \dagger}\right\}\left\{\phi_{0}^{\mu} \otimes \phi_{m}^{\mu}\right\}, \\
N_{12} & =\eta_{\mu \nu} \sum_{n, m=1}^{\infty}\left(n^{2}+m^{2}\right)^{1 / 2}\left\{\phi_{n}^{\mu \dagger} \otimes \phi_{m}^{\mu \dagger}\right\}\left\{\phi_{n}^{\mu} \otimes \phi_{m}^{\mu}\right\}, \\
a & =\eta_{\mu}^{\mu} \sum_{n=1}^{\infty} n, \\
b & =\frac{1}{2} \eta_{\mu}^{\mu} \sum_{n, m=1}^{\infty} \sqrt{n^{2}+m^{2}} .
\end{aligned}
$$

\section{Spectrum}

We will employ the Zeta function regularization scheme to regularize the infinite divergent summation in (17) and (18). The contributions arising from the infinite sum (18) have not been considered carefully in earlier works and have usually been taken as some background field effects.

In fact, there is another scheme [11], the so-called Epstein Zeta functions to regularize the infinite sums of type (18):

$$
\begin{aligned}
\sum_{n, m=1}^{\infty} \sqrt{\left(\frac{n}{c_{1}}\right)^{2}+\left(\frac{m}{c_{2}}\right)^{2}} \\
=\frac{1}{24}\left(\frac{1}{c_{1}}+\frac{1}{c_{2}}\right)-\frac{\zeta(3)}{8 \pi^{2}}\left(\frac{c_{1}}{c_{2}^{2}}+\frac{c_{2}}{c_{1}^{2}}\right) \\
\quad-\frac{\pi^{3 / 2}}{2 \sqrt{c_{1} c_{2}}}\left[\exp \left(-2 \pi \frac{c_{1}}{c_{2}}\right)\left(1+\mathcal{O}\left(10^{-3}\right)\right)\right]
\end{aligned}
$$

with $\zeta(3) \approx 1.202$ and $c_{1} \leq c_{2}$. The infinite sums (17) and (18) appearing in the quantization of the open 2-brane can be written as

$$
\begin{aligned}
& a=\eta_{\mu}^{\mu} \sum_{n=1}^{\infty} n=(D-2) \sum_{n=1}^{\infty} n=(D-2) \zeta(-1), \\
& b=\frac{1}{2} \eta_{\mu}^{\mu} \sum_{n, m=1}^{\infty} \sqrt{n^{2}+m^{2}}=\frac{D-2}{2} \sum_{n, m=1}^{\infty} \sqrt{n^{2}+m^{2}} .
\end{aligned}
$$

Numerically, $a=-2$ while $b \approx 1 / 2$, given that the spacetime dimension $D=26$.

According to the above calculation and the fact that $T_{00}=$ $0=H$, we can rewrite (13), the mass formula of the open $2-$ brane spectrum, as

$$
\alpha^{\prime} M^{2}=N_{1}+N_{2}+N_{12}-\frac{3}{2}
$$

By definition, the ground state of the system is annihilated by the operators $\phi_{n \geqslant 0}^{\mu}$ and $\phi_{m \geqslant 0}^{\mu}$, with the condition that $n \cdot m \neq 0$ :

$$
\phi_{n} \otimes \phi_{m}|0,0\rangle=0 \text {. }
$$

A general state $|\varphi, \chi\rangle$, in the Fock space, can be obtained by application of the creation operators $\phi_{n}^{\mu \dagger}$ and $\phi_{m}^{\mu \dagger}$ on the ground state:

$$
|\varphi, \chi\rangle=\Pi_{n, m}\left\{\phi_{n_{i}}^{\mu \dagger} \otimes \phi_{m_{i}}^{\mu \dagger}\right\}|0,0\rangle
$$

with $\sum_{i} n_{i}=\varphi$ and $\sum_{i} m_{i}=\chi$. The states $|\varphi, \chi\rangle$ and $|\chi, \varphi\rangle$ have the same mass squared.

At the lowest mass level, the number operators $N_{1}, N_{2}$, and $N_{12}$ are zero, so

$$
\alpha^{\prime} M^{2}=-\frac{3}{2}
$$

The first excited level contains two kinds of states corresponding to $n=1, m=0$ and $n=0, m=1$; that is,

$$
\begin{aligned}
& |1,0\rangle=k_{\mu}\left(\phi_{1}^{\mu \dagger} \otimes \phi_{0}^{\mu \dagger}\right)|0,0\rangle, \\
& |0,1\rangle=l_{\mu}\left(\phi_{0}^{\mu \dagger} \otimes \phi_{1}^{\mu \dagger}\right)|0,0\rangle
\end{aligned}
$$

whose mass-square operator reads

$$
\begin{aligned}
& \alpha^{\prime} M_{k}^{2}=N_{1}+N_{2}+N_{12}-\frac{3}{2}=1+0+0-\frac{3}{2}=-\frac{1}{2}, \\
& \alpha^{\prime} M_{l}^{2}=N_{1}+N_{2}+N_{12}-\frac{3}{2}=0+1+0-\frac{3}{2}=-\frac{1}{2} .
\end{aligned}
$$

The ground and first excited states are both tachyonic. However the value of mass squared operator in [6] is an integer, while we show that it is half integer valued. The ground states are scalar states. We have two different kinds of vector states at the first excited state level corresponding to $|1,0\rangle$ and $|0,1\rangle$.

At the second excited level, there are four kinds of tensor states, featured by

$$
\begin{aligned}
& n=0 \\
& m=2, \\
& n=2 \\
& m=0, \\
& n_{1}=n_{2}=1 \\
& m_{1}=m_{2}=0, \\
& n_{1}=n_{2}=0 \\
& m_{1}=m_{2}=1
\end{aligned}
$$

with the creation operators arranging at this level as follows:

$$
\begin{aligned}
& |2,0\rangle_{1}=k_{\mu \nu}\left(\phi_{1}^{\mu \dagger} \otimes \phi_{0}^{\mu \dagger}\right)\left(\phi_{1}^{\nu \dagger} \otimes \phi_{0}^{\nu \dagger}\right)|0,0\rangle, \\
& |2,0\rangle_{2}=k_{\mu}\left(\phi_{2}^{\nu \dagger} \otimes \phi_{0}^{\nu \dagger}\right)|0,0\rangle, \\
& |0,2\rangle_{1}=l_{\mu \nu}\left(\phi_{0}^{\mu \dagger} \otimes \phi_{1}^{\mu \dagger}\right)\left(\phi_{0}^{\nu \dagger} \otimes \phi_{1}^{\nu \dagger}\right)|0,0\rangle, \\
& |0,2\rangle_{2}=l_{\mu}\left(\phi_{0}^{\nu \dagger} \otimes \phi_{2}^{\nu \dagger}\right)|0,0\rangle .
\end{aligned}
$$


For these states, the number operators are $N_{1}=2, N_{2}=$ $N_{12}=0$ or $N_{2}=2, N_{1}=N_{12}=0$. The mass squared operator will become

$$
\begin{aligned}
\alpha^{\prime} M^{2} & =N_{1}+N_{2}+N_{12}-\frac{3}{2} \\
& =0+2+0-\frac{3}{2} \text { or } 2+0+0-\frac{3}{2}=\frac{1}{2} .
\end{aligned}
$$

We can see that the spectrum of the bosonic 2-brane does not contain any massless states to play the role of gravitons. Moreover, the mass squared operator for the open 2-brane only gives half integer mass squared values.

\section{Summary and Conclusion}

In this paper, firstly we review the quantization of open 2brane, starting from the Polyakov action. We then investigate the spectrum of the open 2-brane by taking into account the contributions of both the infinite sums (17) and (18). On the basis of this we can draw the conclusion that there are no gravitons present in the bosonic open 2-brane at the massless level, or there are no massless states in the bosonic open 2brane spectrum. This further implies that the bosonic open 2-brane theory is not a theory of quantum gravity. This is reminiscent of the fact that, in the string case [12], simple open strings do not form self-complete system of quantum gravity.

\section{Conflict of Interests}

The authors declare that there is no conflict of interests regarding the publication of this paper.

\section{Acknowledgments}

The authors thank Jian-Feng Wu for suggesting to them literatures on the special zeta functions [11]. The work is supported by the Open Project Program of State Key Laboratory of Theoretical Physics, Institute of Theoretical Physics, Chinese Academy of Sciences, China. M. Abdul Wasay would also like to thank Yasir Jamil for providing the facilities at Physics Department, University of Agriculture, Faisalabad, where part of this work was carried out.

\section{References}

[1] P. A. M. Dirac, "An extensible model of the electron," Proceedings of the Royal Society of London Series A: Mathematical and Physical Sciences, vol. 268, no. 1332, pp. 57-67, 1962.

[2] J. Hughes, J. Liu, and J. Polchinski, "Supermembranes," Physics Letters B, vol. 180, no. 4, pp. 370-374, 1986.

[3] E. Bergshoeff, E. Sezgin, and P. K. Townsend, "Supermembranes and eleven-dimensional supergravity," Physics Letters B, vol. 189, no. 1-2, pp. 75-78, 1987.

[4] E. Bergshoeff, E. Sezgin, and P. K. Townsend, "Properties of the eleven-dimensional super membrane theory," Annals of Physics, vol. 185, no. 2, pp. 330-368, 1988.

[5] B. de Wit, M. Luscher, and H. Nicolai, "The supermem-brane is unstable," Nuclear Physics B, vol. 320, no. 1, pp. 135-159, 1989.
[6] Y.-C. Huang and C.-X. Yu, "Quantization and spectrum of an open 2-brane," Physical Review D, vol. 75, no. 4, Article ID 044011, 2007.

[7] C.-X. Yu and Y.-C. Huang, "Dirac quantization of open $p$ brane," Physics Letters B, vol. 647, no. 1, pp. 49-55, 2007.

[8] G. T. Horowitz and L. Susskind, "Bosonic M theory," Journal of Mathematical Physics, vol. 42, no. 7, pp. 3152-3160, 2001.

[9] C.-X. Yu, C. Huang, P. Zhang, and Y.-C. Huang, "First and second quantization theories of open p-brane and their spectra," Physics Letters, Section B: Nuclear, Elementary Particle and HighEnergy Physics, vol. 697, no. 4, pp. 378-384, 2011.

[10] R. Banerjee, P. Mukherjee, and A. Saha, "Bosonic P-brane and arnowitt-deser-misner decomposition," Physical Review D, vol. 72, no. 6, Article ID 066015, 2005.

[11] E. Elizalde, Ten Physical Applications of Spectral Zeta Functions, Springer, Berlin, Germany, 2nd edition, 2012.

[12] J. Polchinski, String Theory, vol. 1, Cambridge University Press, Cambridge, UK, 2001. 

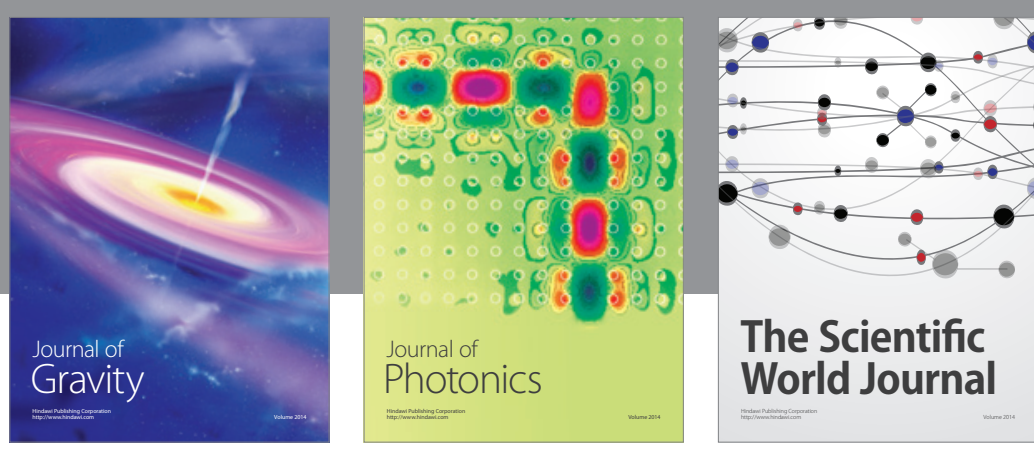

The Scientific World Journal
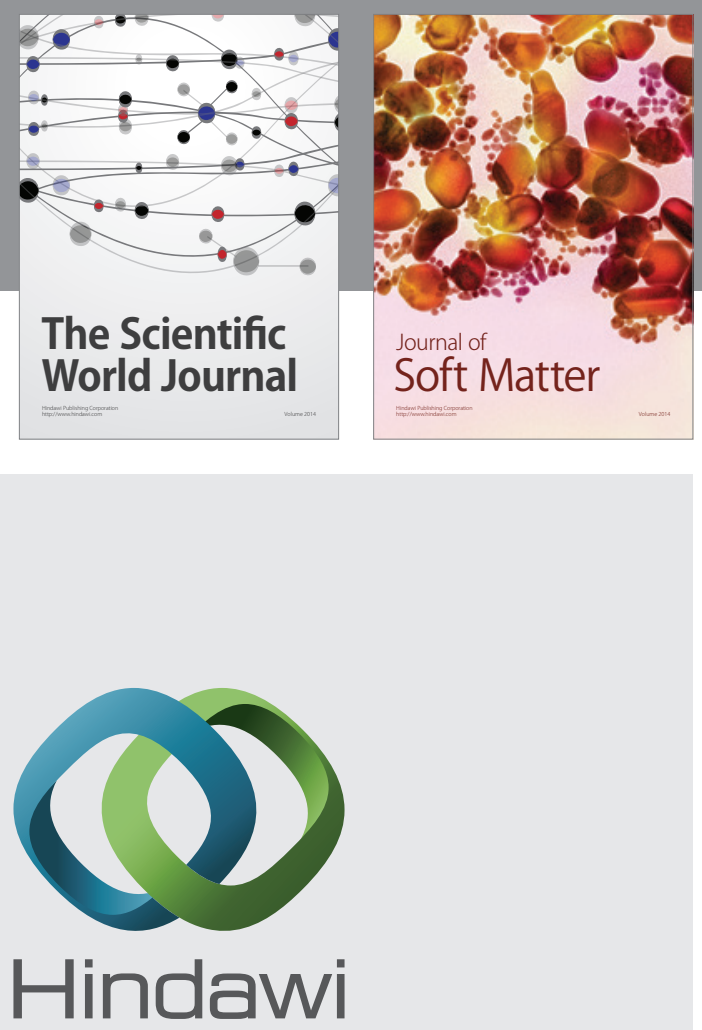

Submit your manuscripts at

http://www.hindawi.com

nternational Journal of

Statistical Mechanics
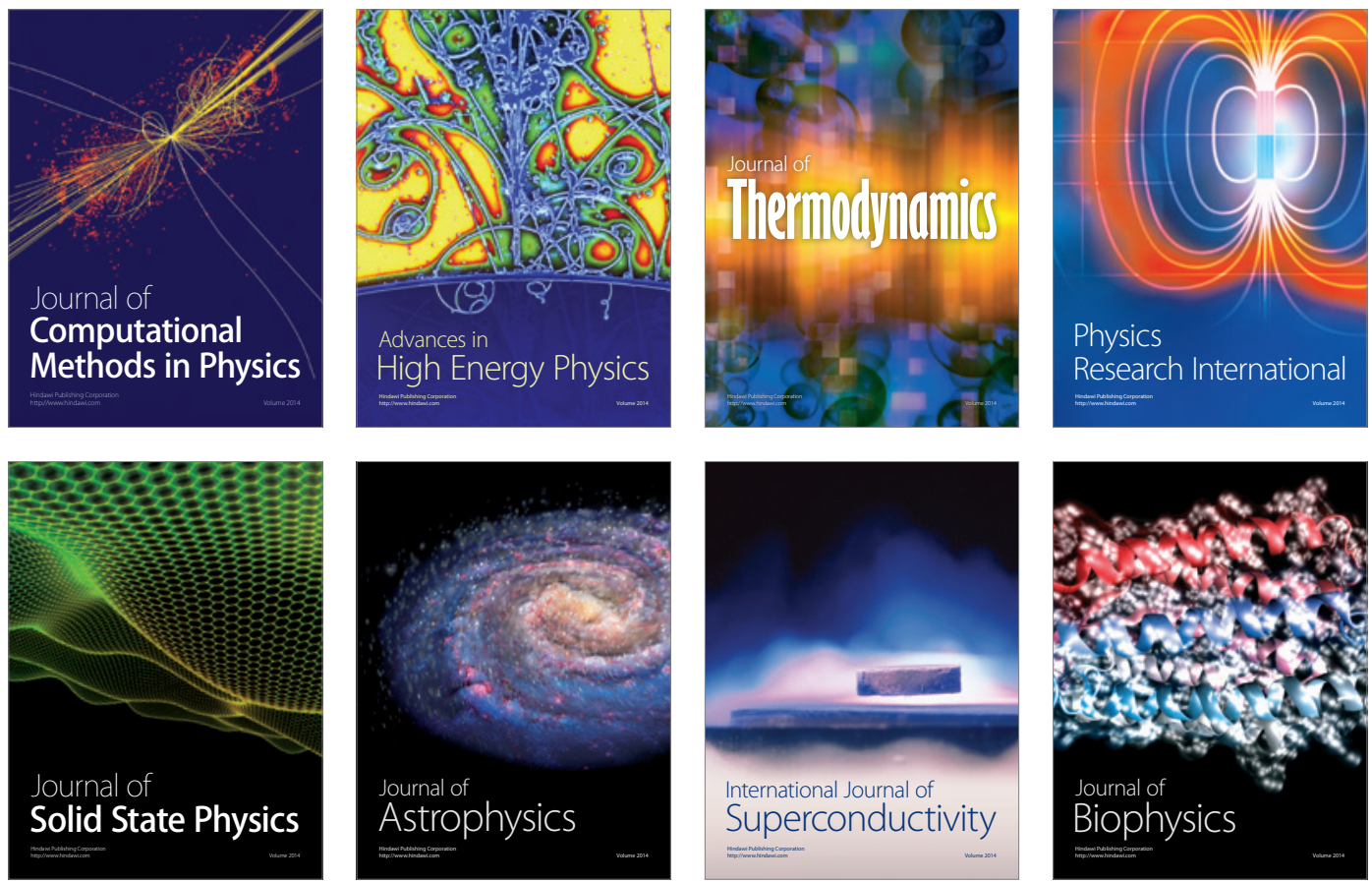
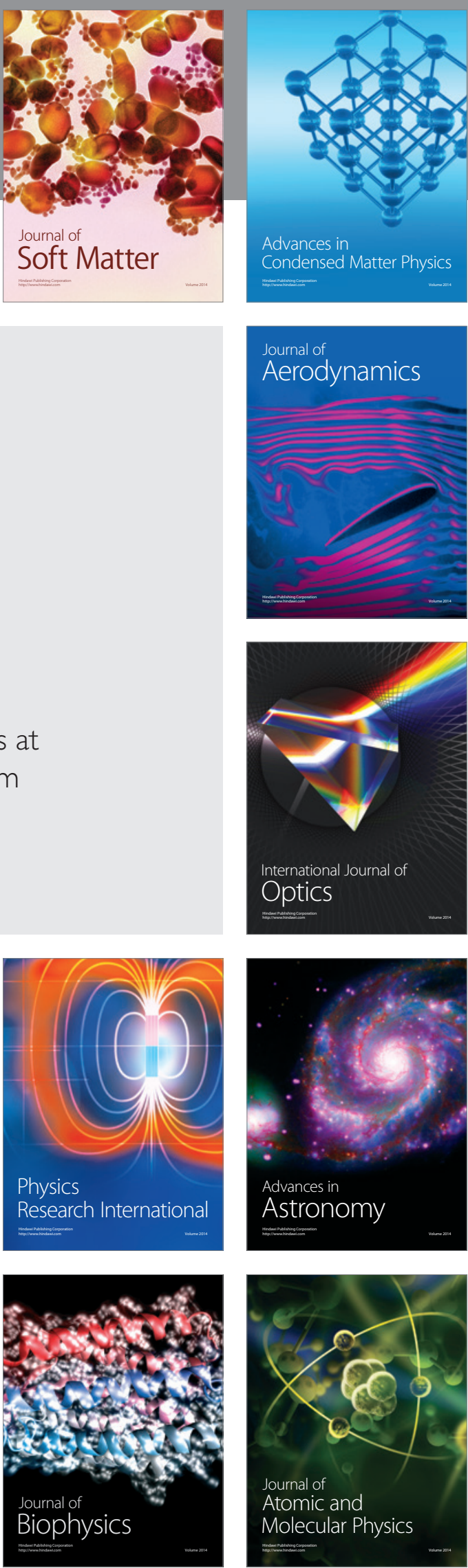